\title{
Impact of State Anxiety, Mindfulness on Cardiac Autonomic Variables in Healthy Adults: A Correlational Study
}

\author{
Jintu Kurian ${ }^{1, *}$, and Raghvendra Bhat ${ }^{2}$ \\ ${ }^{1}$ PhD Scholar, Division of Yoga and Life Sciences, S VYASA University, Bangalore, India \\ ${ }^{2}$ Associate Professor, Division of Yoga and Life Sciences, S VYASA University, Bangalore, India
}

Received: 08 Aug, 2019 | Accepted: 30 Aug, 2019 | Published: 05 Sep, 2019

*Corresponding author: Kurian J, PhD Scholar, Division of Yoga and Life Sciences, S VYASA University, Bangalore, India, Tel: $9606152928 ;$ E-mail: jintukurian@gmail.com

Citation: Kurian J, Bhat R (2019) Impact of State Anxiety, Mindfulness on Cardiac Autonomic Variables in Healthy Adults: A Correlational Study. J Hear Health 5(2): dx.doi.org/10.16966/2379-769X.150

Copyright: (c) 2019 Kurian J, et al. This is an open-access article distributed under the terms of the Creative Commons Attribution License, which permits unrestricted use, distribution, and reproduction in any medium, provided the original author and source are credited.

\begin{abstract}
The interest about mindfulness has been increased in the last decades. The epidemiological studies shows a hike from communicable diseases to non-communicable diseases indicating that the adults are more prone for anxiety related, mindfulness related and autonomic variables like heart rate, heart rate variability and related issues. State anxiety is a topic of discussion among all age groups and the way it reflects on sympathetic and parasympathetic nervous system is also eye catchy in the field of psycho physiological research. This study tired to understand the impact of state anxiety and mindfulness on cardiac autonomic regulation on heart rate variability. Eighty three subjects were assessed using state anxiety and mindfulness questionnaire and heart rate variability measurement in Biopac equipment. The result showed a negative correlation between mindfulness and state anxiety $(r=-.942, P<0.001)$. State anxiety increases sympathetic activity $(P<0.05)$ and decreases parasympathetic activity of Autonomic Nervous System $(P<0.05)$. Whereas, mindfulness increases parasympathetic activity $(P<0.05)$ and decreases sympathetic tone $(P<0.05)$. The study could bring up the importance of improving the mindfulness, bringing down the anxiety, and there by balancing cardiac nerves of sympathetic and parasympathetic nervous system.
\end{abstract}

Keywords: Mindfulness; State anxiety; Heart rate variability; Cardiac Autonomic Regulation

Abbreviations: SMAAS: State Mindful Attention Awareness Scale; STAI: State Anxiety Inventory; HRV: Heart Rate Variability; LF: Low Frequency; HF: High Frequency; ANS: Autonomic Nervous System

\section{Introduction}

Epidemiological studies show that adults are prone for anxiety related [1], mindfulness related [2] and autonomic variables like heart rate, heart rate variability [3] related issues. $24.4 \%$ adults in India are found with anxiety related issues. Around the world $65-70 \%$ of adults are found to be undergoing anxiety disorders [4]. State Anxiety (SA) is feeling anxious, experiencing a threat to an existing goal at the present moment. SA results in getting deprived of awareness on the present moment and activities [5]. Fortunately, the remedy for these emerging issues is in near reach. Therapies like mindfulness based stress reduction and training mind with focusing and defocusing techniques are gaining attention with its impact on gaining emotional stability and relieving anxiety [6].

Mindfulness is defined as the awareness emerging through paying attention on the present moment, or on the purpose, being nonjudgmental to the opening up of moment to moment experiences [7]. It is the experience of the present moment and is being widely applied in clinical psychology. The understanding of mindfulness has got its origin from Buddhist traditions [8]. It is gaining popularity worldwide as being found effective in dealing with clinical interventions. Mindfulness was introduced by Crane RS, et al. [9] as a therapeutic practice and is practiced as a stress reduction way known as Mindful Based Stress Reduction (MBSR) and Mindfulness-Based Cognitive Therapy (MBCT) as therapeutic ways of management of stress and cognitive impairment. Mindfulness-Based Stress Reduction (MBSR) [10] is a systematic training in meditation that enables people to self-regulate their reactions to stress and the negative emotion [11]. Mindfulness acts as a protection against emotional exhaustion [12]. Mindfulness is an attribute of consciousness long believed to uplift the awareness in daily living activities [13]. State anxiety can be conceptualized as a response to a particular anxiety provoking stimulus. State anxiety is the anxiety being felt at the present moment of time [14]. It occurs as a response to a definite situation. Situationspecific anxiety has been used to emphasize the multifaceted and persistent nature of some anxieties [15]. It is usually aroused at specific situations, such as public speaking, class Test [16]. 
Heart Rate Variability (HRV) refers to beat to beat alterations in the heart rate and is associated with cardiac autonomic regulation. It is a vagal nerve-mediated biomarker of autonomic variables, showing cardiac function used to investigate chronic illness, psychopathology, stress and attention-regulation processes such as meditation [17]. HRV shows the function of the heart and influences of autonomic and other physiological systems on HRV [18]. The measurement of $\mathrm{HRV}$ is recorded using ambulatory Electrocardiograph (ECG) and is measured by linear, non-linear methods, and various other methods [19]. HRV analysis in clinical cardiology is its measurement in post infraction patients. Baroreceptors, sensory mechanoreceptors, and chemoreceptors are located throughout the large vessels. The peripheral vasculature and the ventricles of the heart modulate the low frequency and very low frequency oscillations of heart rate [20]. Mindfulness has been incorporated into several treatment approaches for psychopathology. Despite the popularity of this approach, relatively few empirical investigations have examined the relationship between mindfulness and autonomic indicators of flexible emotion regulation, such as HRV.

\section{Rationale of the Study}

Research studies have shown that HRV may vary with mental states [21]. Studies promote mindfulness based therapies for anxiety disorders [22]. The association between mindfulness, state anxiety and HRV were not studied. Hence, in the present study, we have assessed correlation between mindfulness, state anxiety and heart rate variability in healthy adults. This study focused on understanding the need to decrease anxiety, increase mindfulness and thereby to bring an autonomic balance in between cardiac branches of sympathetic and parasympathetic nervous system.

\section{Aim of the Study}

The aim of this study was to test the level of relationship between the mindfulness, state anxiety and HRV in healthy volunteers.

\section{Methods and Participants}

Announcement about the study was placed on the University notice board of YIC batch of Swami Vivekananda Yoga Anusandhana Samsthana (S VYASA) in August 2016. Eighty-three (37 male and 46 female) healthy volunteers with their age ranging from 28 to 50 years (mean age \pm SD; $33.42 \pm 5.63$ years) participated in the study. They were naive yoga students joined for one month yoga instructor course of S-VYASA, Bengaluru, India. Participants who could read and understand english were included for the study. Participants with any kind of illness and who were on medication were excluded from the study. Participants with age above fifty years were excluded because HRV is known to vary with age. The study protocol was explained to all subjects and signed informed consent was obtained. Correlational study design was used.

State Mindful Attention Awareness scale (SMAAS) is a valid tool for measuring state mindfulness. The scale is designed to assess the shortterm or current expression of a core characteristic of mindfulness. This is a receptive state of mind and sensitive awareness of observing the present moment. The SMAAS draws items drawn from the trait form of the MAAS (e.g., "I'm finding it difficult to stay focused on what's happening in the present"). SMAAS has shown excellent psychometric properties (Cronbach's alpha $=92$ ).

The State Trait Anxiety Inventory-Short Form (STAI-SF) consists of two questionnaires of 20 items each. The first questionnaire measures state anxiety (how one feels at the moment); the second, trait anxiety (how one generally feels). A standardized, short-form of STAI has been used for this study. STAI-SF consists of six items assessing the extent to which patients feel "calm," "'tense," "'upset," "relaxed," "content," and "worried" on a 4-point scale ranging from "not at all" to "very much." Items consist of equal numbers of anxiety-present and anxiety-absent. Three items are scored in reverse order to avoid a response bias. The items were summed to produce a total score in which higher scores are related to greater anxiety. The six items STAI-SF demonstrated good reliability coefficient $(r>0.82)$.

Heart Rate Variability (HRV) was recorded using 16 channel human physiology system (Power Lab 16/35, AD Instruments, Australia) with a standard bipolar limb lead I configuration. The ECG was digitized using a 16-bit analog-to-digital converter at a sampling rate of $1 \mathrm{KHz}$ and was analyzed off-line to obtain the HRV spectrum. Frequency domain analysis of HRV data were carried out.

\section{Data collection and Statistical Analysis}

HRV was recorded in empty stomach, between 6:30 am to 8:30 am on 3 consecutive days. Protocol about the study and mode of recording was explained to 109 students of YIC course and 96 volunteered in the study. Out of which 13 couldn't meet up the inclusion criteria and data was acquired from 83 of the volunteers. Subjects were given instructions to have a good night sleep on the previous day of recording. Before recording, subjects were seated in the cushioned chair comfortably attaching the 3 leads (right arm, left arm and right ankle) and 5 minutes preparation time was allotted to adjust themselves with the situation of recording room. The room was airconditioned $\left(\right.$ at $\left.20^{\circ} \mathrm{C}\right)$. After 5 minutes, breath rate was observed and if the subject was found still with a high respiration rate, resting time was extended to another 5 minutes and recording was taking for a duration of 5 minutes. Subjects were allowed to sit by keeping eyes closed and/ or to watch the data run live.

Recording of HRV was monitored by the technician throughout the recording. Extraction of HRV data was done using Kubios software. Signal filtering was done by deleting the portion of data with noise in the form of artifacts using AcqKnowledge Software. Data with huge artifacts were re-recorded. 13 dropouts were based on the error in the acquired data. The subjects were given questionnaires before the HRV data recording to fill up questions to access state anxiety and mindfulness.

Data were entered and tabulated in excel. It was analyzed using the statistical analysis software R (version 3.2). Data were tested for normality using Kolmogorov Smirnov test. Pearson's correlation was used to understand relationship between above mentioned variables. Pearson's correlation test was used to understand the relationship between SMAAS, state anxiety and components of heart rate variability.

\section{Results}

Significant negative correlation was found between SMAAS and STAI $(\mathrm{r}=-0.942, \mathrm{P}<0.001)$ (Table 1$)$. This indicates that when there is an increase in state anxiety, mindfulness reduces, hampering the awareness in activities. Also, the result shows that when there was an increase in state anxiety, sympathetic activity increased as shown by a positive correlation between STAI and LF of HRV ( $r=0.252$, $\mathrm{P}<0.05$ ). State anxiety was found to be negatively correlated with parasympathetic activity $(\mathrm{r}=-0.255, \mathrm{P}<0.05)$. On the other hand there is a decrease in sympathetic tone and an increase in parasympathetic tone found with an increased mindfulness level as indicated by a negative correlation between SMAAS and LF $(r=-0.259, \mathrm{P}<0.05)$ and 
Table 1: Mean and standard deviation of variables.

\begin{tabular}{|l|c|c|c|c|}
\hline \multicolumn{1}{|c|}{ Variables } & SMAAS & STAl & LF & HF/HF ratio \\
\hline $\begin{array}{l}\text { Scores } \\
\text { (mean } \pm \text { SD) }\end{array}$ & $3.78 \pm 1.26$ & $12.81 \pm 3.61$ & $56.98 \pm 20.02$ & $42.78 \pm 19.93$ \\
\hline
\end{tabular}

SMAAS: State Mindful Attention Awareness Scale, STAI: State Anxiety Inventory, LF: Low Frequency, HF: High Frequency

Table 2: Correlation between SMAAS, STAI and HRV.

\begin{tabular}{|c|c|c|c|c|c|c|}
\hline & & STAI & SMAAS & LF nu & HF nu & LF/HF ratio \\
\hline \multirow{2}{*}{ STAI } & Pearson Correlation & 1 & $-0.942^{* *}$ & $0.252^{*}$ & $-0.255^{*}$ & $0.304^{* *}$ \\
\hline & Sig. (2-tailed) N=83 & & 0.000 & 0.022 & 0.020 & 0.005 \\
\hline \multirow{2}{*}{ SMAAS } & Pearson Correlation & $-0.942^{* *}$ & 1 & $-0.259^{*}$ & $0.264^{*}$ & $-0.313^{* *}$ \\
\hline & Sig. (2-tailed) N=83 & 0.000 & & 0.018 & 0.016 & 0.004 \\
\hline \multirow{2}{*}{ LF nu } & Pearson Correlation & $0.252^{*}$ & $-0.259^{*}$ & 1 & $-0.998^{* *}$ & $0.771^{* *}$ \\
\hline & Sig. (2-tailed) N=83 & 0.022 & 0.018 & & 0.000 & 0.000 \\
\hline \multirow{2}{*}{ HF nu } & Pearson Correlation & $-0.255^{*}$ & $0.264^{*}$ & $-0.998^{* *}$ & 1 & $-0.773^{* *}$ \\
\hline & Sig. (2-tailed) N=83 & 0.020 & 0.016 & 0.000 & & 0.000 \\
\hline \multirow{2}{*}{ LF/HF ratio } & Pearson Correlation & $0.304^{* *}$ & $-0.313^{* *}$ & $0.771^{* *}$ & $-0.773^{* *}$ & 1 \\
\hline & Sig. (2-tailed) N=83 & 0.005 & 0.004 & 0.000 & 0.000 & \\
\hline
\end{tabular}

${ }^{* *}$ Correlation is significant at the 0.01 level (2-tailed).

${ }^{*}$ Correlation is significant at the 0.05 level (2-tailed).

LF: Low Frequency; HF: High Frequency; STAI: State anxiety Inventory; SMAAS: State Mindful Attention Awareness Scale

a positive correlation between SMAAS and HF $(r=-0.264, \mathrm{P}<0.05)$. And also, there was a significant moderate positive correlation between STAI and LF/HF ratio $(\mathrm{r}=0.304, \mathrm{P}<0.05)$ and negative correlation between SMAAS and LF/HF ratio $(\mathrm{r}=-0.313, \mathrm{P}<0.05)$ (Table 2). The correlational score was not found to be appreciable, even though the significance of the result was understood from the obtained P-value.

\section{Discussion}

Previous studies spotlight the link between HRV and emotional upsurges like anger and anxiety [23]. There are studies showing the importance of different meditation techniques on heart rate variability highlighting the importance of keeping oneself aware at the same time relaxed with mindfulness at situations leading to anxiety [24-26]. Trait anxiety reduces HRV and vagal tone which is important risk factor and predictor of cardiovascular morbidity and mortality [21].

Abnormal HRV indicates an increased chance for cardiac problems $[19,27]$. High Frequency (HF) oscillations in HRV are related to respiration and parasympathetic nervous system. It provides information about the phasic efferent vagal input to the heart. The High frequency oscillation of heart rate is modulated mainly by the central nervous system and pulmonary afferent stimuli.

Abnormal readings of HRV can lead to disturbed function at brain and mind level resulting in disorders and diseases at body level. At the brain level the hike in cardiac sympathetic nerve activity can result in sleep disturbances, inability to relax mind and body resulting in an increase in the stress-anxiety levels. Thus we could come into a summary that, anxiety not only increases sympathetic activity but reduces overall heart rate variability. The mechanism by which the HRV varies with mindfulness and anxiety is understood with the function of vagus nerve [28]. Poor signal from the vagus nerve on cardiac vascular system reduces heart rate variability. Healthy heart rate variability is understood to be essential for a healthy heart and in turn for an optimum physiological status.
Result of our study could bring up the impact of mindfulness in increasing parasympathetic activity, by reducing sympathetic tone in adults. The study could highlight that, as state anxiety increases sympathetic tone, there is a reduction in parasympathetic tone. Whereas, LF/HF ratio was found to be high in increased state anxiety level and on the other hand, there was a decrease in LF/HF ratio found with an increased mindfulness. As heart rate variability is important in balancing activity level at both mind and body, it is highly essential to reduce state anxiety and to improve the mindfulness.

\section{Conclusion}

The research study could bring up a clear understanding about the importance of mindfulness in keeping a balance in autonomic variables. Reducing state anxiety is highly important to lower the sympathetic tone and to bring up parasympathetic tone. This is understood by correlation between mindfulness, state anxiety and heart rate variability among adults of the current generation. The stress and anxiety rich days makes the adults deprived of awareness and attention in their activities and this is found to affect the heart rate variability. Hence need for relaxing oneself with available source of therapies like yoga, meditation is pointed out to be highly required to improve the mindfulness to have a healthy journey ahead.

\section{Acknowledgement}

YIC coordinator, Yoga Instructors course department, S VYASA University, Bangalore, India.

All the students of August 2016 YIC batch, who volunteered to join into this research study.

\section{Financial Support}

No financial support was obtained.

\section{Conflict of Interest}

No conflicts of interest. 


\section{References}

1. Bryant C, Jackson H, Ames D (2008) The Prevalence of Anxiety in Older Adults: Methodological Issues and a Review of the Literature. J Affect Disord 109: 233-250.

2. Shorey RC, Brasfield H, Anderson S, Stuart GL (2013) Mindfulness Deficits in a Sample of Substance Abuse Treatment Seeking Adults: A Descriptive Investigation. J Subst Use 19: 194-198.

3. Koskinen $T$, Kähönen M, Jula A, Laitinen T, Keltikangas-Järvinen $L$, et al. (2009) Short-Term Heart Rate Variability in Healthy Young Adults: The Cardiovascular Risk in Young Finns Study. Auton Neurosci 145: 81-88.

4. Sahoo S, Khess CR (2010) Prevalence of Depression, Anxiety, and Stress among Young Male Adults in India: A Dimensional and Categorical Diagnoses-Based Study. J Nerv Ment Dis 198: 901-904.

5. Bishop S, Duncan J, Brett M, Lawrence AD (2004) Prefrontal Cortical Function and Anxiety: Controlling Attention to Threat-Related Stimuli. Nat Neurosci 7: 184-188.

6. Orfus S (2008) The Effect Test Anxiety and Time Pressure on Performance. The Huron University College Journal of Learning and Motivation 46: 118-133.

7. Davidson RJ, Kabat-Zinn J, Schumacher J, Rosenkranz M, Muller D (2003) Alterations in Brain and Immune Function Produced by Mindfulness Meditation. Psychosom Med 65: 564-570.

8. Kang C, Whittingham K (2010) Mindfulness: A Dialogue between Buddhism and Clinical Psychology. Mindfulness 1: 161-173.

9. Crane RS, Brewer J, Feldman C, Kabat-Zinn J, Santorelli S, et al. (2017) What Defines Mindfulness-Based Programs? The Warp and the Weft. Psychol Med 47: 990-999.

10. Ma SH, Teasdale JD (2004) Mindfulness-Based Cognitive Therapy for Depression: Replication and Exploration of Differential Relapse Prevention Effects. J Consult Clin Psychol 72: 31-40.

11. Grossman P, Niemann L, Schmidt S, Walach H (2004) MindfulnessBased Stress Reduction and Health Benefits: A Meta-Analysis. J Psychosom Res 57: 35-43.

12. Escudero-Pérez $\mathrm{S}$, León-Palacios MG, Úbeda-Gómez J, BarrosAlbarrán MD, López-Jiménez AM, et al. (2016) Dissociation and Mindfulness in Patients with Auditory Verbal Hallucinations. J Trauma Dissociation 17: 294-306.

13. Taylor NZ, Millear PMR (2016) The Contribution of Mindfulness to Predicting Burnout in the Workplace. Personality Individual Diff 89: 123-128.

14. Mikolasek M, Berg J, Witt CM, Barth J (2018) Effectiveness of Mindfulness- and Relaxation-Based eHealth Interventions for Patients with Medical Conditions: A Systematic Review and Synthesis. Int J Behav Med 25: 1-16.

15. Martens R, Burton D, Vealey RS, Bump LA, Smith DE (1990) Development and Validation of the Competitive State Anxiety
Inventory-2 (CSAI-2). In: Martens R, Vealey RS, Burton D (eds) Competitive Anxiety in Sport. Human Kinetics Books, Champaign 117-190.

16. Bishop SJ, Duncan J, Lawrence AD (2004) State Anxiety Modulation of the Amygdala Response to Unattended Threat-Related Stimuli. J Neurosci 24: 10364-10368.

17. Krygier JR, Heathers JA, Shahrestani S, Abbott M, Gross JJ, et al. (2013) Mindfulness Meditation, Well-Being, and Heart Rate Variability: A Preliminary Investigation into the Impact of Intensive Vipassana Meditation. Int J Psychophysiol 89: 305-313.

18. Valkama JO, Huikuri HV, Koistinen MJ, Yli-Mäyry S, Airaksinen KE, et al. (1995) Relation between Heart Rate Variability and Spontaneous and Induced Ventricular Arrhythmias in Patients with Coronary Artery Disease. J Am Coll Cardiol 25: 437-443.

19. Wells R1, Outhred T, Heathers JA, Quintana DS, Kemp AH (2012) Matter Over Mind: A Randomized-Controlled Trial of Single-Session Biofeedback Training on Performance Anxiety and Heart Rate Variability in Musicians. PloS One 7: e46597.

20. Tyagi A, Cohen M, Reece J, Telles S, Jones L (2016) Heart Rate Variability, Flow, Mood and Mental Stress During Yoga Practices in Yoga Practitioners, Non-Yoga Practitioners and People with Metabolic Syndrome. Appl Psychophysiol Biofeedback 41: 381-393.

21. Quintana DS, Guastella AJ, Outhred T, Hickie IB, Kemp AH (2012) Heart Rate Variability is Associated with Emotion Recognition: Direct Evidence For A Relationship Between The Autonomic Nervous System and Social Cognition. Int J Psychophysiol 86: 168-172.

22. Mankus AM, Aldao A, Kerns C, Mayville EW, Mennin DS (2013) Mindfulness and Heart Rate Variability in Individuals with High and Low Generalized Anxiety Symptoms. Behav Res Ther 51: 386-391.

23. Phongsuphap S, Pongsupap Y, Chandanamattha P, Lursinsap C (2008) Changes in Heart Rate Variability During Concentration Meditation. Int J Cardiol 130: 481-484.

24. Miu AC, Heilman RM, Miclea M (2009) Reduced Heart Rate Variability and Vagal Tone in Anxiety: Trait versus State, and the Effects of Autogenic Training. Auton Neurosci 145: 99-103.

25. Helmes E, Ward BG (2017) Mindfulness-Based Cognitive Therapy for Anxiety Symptoms in Older Adults in Residential Care. Aging Ment Health 21: 272-278.

26. Hoshiyama M, Hoshiyama A (2008) Heart Rate Variability Associated with Experienced Zen Meditation. Computers in Cardiology 35: 569572.

27. Phongsuphap S, Pongsupap $Y$ (2011) Analysis of Heart Rate Variability During Meditation by a Pattern Recognition Method. 2011 Computing in Cardiology 2011: 197-200.

28. Huikuri HV, Koistinen MJ, Yli-Mäyry S, Airaksinen KE, Seppänen T, et al. (1995) Impaired Low-Frequency Oscillations of Heart Rate in Patients with Prior Acute Myocardial Infarction and Life-Threatening Arrhythmias. Am J Cardiol 76: 56-60. 